\title{
Análisis descriptivo de Entornos Personales de Aprendizaje: estudio de caso en Enseñanza Obligatoria
}

\author{
Descriptive analysis of PLEs: a case study in Compulsory Education
}

\author{
Alberto Jiménez Hidalgo \\ Universidad de Murcia \\ alb.jim@hotmail.com
}

Recibido: 20/03/2019

Aceptado: 05/12/2019

Publicado: 01/06/2020

\section{RESUMEN}

Existe en la actualidad la necesidad de explicar cómo aprendemos en contextos de educación formal, no formal e informal, de forma autónoma y apoyándonos en los recursos tecnológicos a nuestro alcance. Los Entornos Personales de Aprendizaje (PLE por sus siglas en inglés, Personal Learning Environment) son un constructo teórico en torno al aprendizaje que ha recibido la atención de la comunidad investigadora y educativa durante los últimos años y que ha intentado dar respuesta a esta necesidad. Los estudios de tipo práctico sobre el PLE son menos frecuentes que los de tipo teórico, por lo que es necesario complementar el corpus de investigaciones sobre PLE con trabajos que recojan datos empíricos.

En esta línea presentamos este estudio de caso, diseñado bajo un enfoque cuantitativo de investigación y en el que llevamos a cabo el análisis descriptivo del PLE de alumnos desde $5 .^{\circ}$ de Primaria hasta $4 .^{\circ}$ de ESO de un centro escolar, con el objetivo de utilizar las conclusiones para poder elaborar un plan de actuación y mejora de las estrategias docentes.

Se utilizó un cuestionario ad hoc y recogimos datos de 188 estudiantes. Las principales conclusiones a las que llegamos son las siguientes: la muestra analizada dice conocer y aplicar por igual todas las dimensiones de su PLE, pero lo hace de forma limitada y superficial; un factor determinante para el éxito en este tipo de proyectos es la cantidad de dispositivos disponibles, y no tanto la tipología de los mismos; la edad de los alumnos de Primaria no es impedimento para trabajar sobre sus propios PLE; los estudiantes se sienten más cómodos trabajando de forma tradicional que de formas más innovadoras; por último, la dimensión sobre la que los alumnos han demostrado tener menos conocimientos es la referente a compartir y comunicarse en red.

\section{PALABRAS CLAVE}

Entorno personal de aprendizaje; estudio de caso; Educación Primaria; Educación Secundaria.

\section{ABSTRACT}

There is nowadays a need to explain how we learn in formal, non-formal and informal contexts, in autonomous ways and supported by the available technological resources. Personal Learning Environments (PLEs) are a new theoretical construct about learning that has received the attention of both researchers and educators in the last few years and that tries to give an answer to this issue. Applied studies about PLEs are much less frequent than theoretical ones; the PLE research corpus needs to be complemented with a number of works that give some insight on practical examples of PLEs.

In this field we present this case study, designed following the quantitative approach of investigation and where we show the descriptive analysis of PLEs of students from $5^{\text {th }}$ Grade in Elementary School up to $4^{\text {th }}$ Grade in Secondary School, with the aim to provide the school Administrators with a comprehensive action plan to improve teaching strategies. 
An ad hoc questionnaire was used and we collected data from 188 students. The main conclusions we have reached are the following: the sample analyzed claims to know and apply all the dimensions of their PLE equally, although this is done in a limited and superficial way; a key factor for the success of these kind of projects is the quantity of devices available, and not so much what type these are; age is not a problem for Elementary School students to work on their own PLEs; students feel more comfortable working traditionally rather than in more innovative ways; and last, the dimension related to sharing and communicating online for learning is the least developed one in these students' PLEs.

\section{KEYWORDS}

Personal learning environment; case study; Primary Education; Secondary Education.

\section{CITA RECOMENDADA}

Jiménez, A. (2020). Análisis descriptivo de Entornos Personales de Aprendizaje: estudio de caso en Enseñanza Obligatoria. Revista Interuniversitaria de Investigación en Tecnología Educativa, 8, 1-15. https://doi.org/10.6018/riite.369311

\section{Principales aportaciones del artículo y futuras líneas de investigación:}

- Análisis comparativo de las principales experiencias prácticas que se han publicado en torno al PLE de los alumnos en Enseñanza Obligatoria.

- Descripción analítica del PLE de alumnos desde 5. de Primaria hasta $4 .^{\circ}$ de ESO, así como de las diferentes partes que lo forman.

- Propuesta de plan de actuación para el centro escolar sobre el que se ha investigado.

\section{INTRODUCCIÓN Y ESTADO DEL ARTE}

El interés que los entornos personales de aprendizaje (o PLE, Personal Learning Environment) han recibido en los últimos años queda demostrado por hechos como la amplia cantidad de artículos y números monográficos publicados, los encuentros, congresos y proyectos de investigación centrados en el tema, o la amplia oferta de recursos sobre PLE existente en red.

Esto contrasta con la escasez en la cantidad de investigaciones de tipo aplicado sobre el tema (Benito, 2016). En la producción de tipo teórico distinguimos dos grupos de autores: los que entienden el PLE desde una perspectiva más instrumental, y los que lo entienden desde una perspectiva más cercana a la filosofía del aprendizaje (Castañeda \& Adell, 2013). Incluso se ha intentado poner en práctica la idea de PLE desde la óptica institucional mediante el PLE institucional o iPLE (Salinas et al., 2011). Existe además cierto consenso en que se pueden distinguir varios componentes dentro del PLE, siendo generalmente reconocidas una parte de consumo de información, una parte de creación de productos y una parte de socialización para el aprendizaje (Attwell, 2007; Milligan, 2006; Dabbagh \& Kitsantas, 2012; Peña, 2013). Cada una de estas dimensiones se manifiesta mediante categorías y subcategorías como son las fuentes de información, herramientas, mecanismos, actividades, actitudes y aptitudes del individuo (Castañeda \& Adell, 2013). También se ha investigado de manera teórica la manera en la que el PLE se puede relacionar fácilmente con teorías del aprendizaje tradicionales como el constructivismo social (van Harmelen, 2006) o con otras más nuevas como la heutagogía, la peeragogy, el conectivismo o el aprendizaje rizomático (Wheeler, 2012).

En cuanto a experiencias con ejemplos reales de PLE de alumnos de Primaria y Secundaria en acción, destacan el Proyecto Goldau en Suiza (Döbeli \& Neff, 2010), el Campus SAPO en Portugal (Pais et al., 2013), la experiencia en la Escuela Amadeus en Países Bajos (Rahimi et al., 2012), y otras del ámbito estatal como son el Proyecto PIPLEP en Castilla - La Mancha (Nieto \& Dondarza, 2016) o el Proyecto Huerto Digital en Cataluña (Martín \& Torres, 2010) entre otras. Las investigaciones aplicadas sobre el PLE de alumnos universitarios son ligeramente más numerosas pero todavía igualmente escasas, destacando el Proyecto Dipro 2.0 (Cabero, 2014), el Proyecto CAPPLE (Prendes \& Román, 2017; Prendes et al., 2019) y las 
experiencias de uso conjunto de PLE y Recursos Educativos en Abierto (REA) que encontramos en Vázquez et al. (2016). Así pues, nos encontramos ante la necesidad de experimentar con ejemplos de PLE reales en diferentes contextos de aprendizaje (Attwell et al., en Castañeda et al., 2016).

Este artículo, el cual presenta los resultados de la investigación realizada en el Máster Interuniversitario en e-learning y gestión del conocimiento, pretende contribuir en este sentido a ampliar la literatura sobre casos prácticos de PLE. Para ello hemos diseñado un estudio de caso que, bajo un enfoque de investigación cuantitativo, un diseño transversal y descriptivo y mediante el uso del cuestionario como herramienta de recogida de la información, pretende dar respuesta a la siguiente pregunta: ¿Cómo es el PLE de los alumnos de Primaria y Secundaria del centro educativo seleccionado?

En cualquier caso, queda patente la necesidad de seguir avanzando en la investigación con ejemplos de PLE reales y en acción.

\section{MÉTODO}

Este proyecto es un estudio transversal, de tipo descriptivo y con diseño de estudio de caso. La metodología de investigación utilizada es de tipo cuantitativo; se recogió la información de los alumnos mediante cuestionarios creados ad hoc y se aplicaron diferentes técnicas de análisis de los datos.

\subsection{OBJETIVOS DEL PROYECTO}

Con el fin de dar respuesta a la pregunta de investigación, hemos planteado los siguientes objetivos:

1. Analizar la percepción que los estudiantes desde $5 .$. de Primaria hasta $4 . . \circ$ de ESO tienen sobre su propio PLE.

2. Diseñar y proponer al centro educativo un plan de actuación a partir de la detección de los aspectos menos desarrollados en el PLE de estos alumnos y con el objetivo de mejorarlos.

\subsection{CONTEXTO}

El contexto donde se aplica este trabajo es un centro privado y laico situado en Albacete (España) y que cubre las etapas educativas desde Educación Infantil hasta Bachillerato. Este centro es el único de sus características en la ciudad y uno de los pocos en su comunidad. Sus alumnos provienen de entornos familiares de nivel adquisitivo alto, especialmente los de los cursos de Primaria. En los últimos años el centro ha implementado una serie de cambios en su gestión, entre los que destaca la creación de la figura del Coordinador TIC y la ampliación y mejora de la red wifi.

Cabe destacar que este trabajo es el primer contacto que desde el centro se tiene con el PLE, tanto desde un punto de vista teórico como aplicado.

\subsection{PARTICIPANTES}

El contexto de la investigación fue elegido por conveniencia, la población son los alumnos del centro escolar y la muestra es de tipo no probabilístico elegida igualmente por conveniencia (por cercanía al investigador y facilidad de acceso). La muestra la forman los alumnos desde 5.․ de Primaria hasta 4. de ESO $(n=188)$. El cuestionario se aplicó en horario lectivo a todos los alumnos de los cursos seleccionados presentes ese día en las aulas.

Los grupos analizados presentan algunas diferencias socioeconómicas que creemos debemos destacar. Los grupos de Primaria llevan 7 años cada uno en la escuela, son alumnos de familias especialmente pudientes y tienen bien asimilada la cultura de centro. Los grupos de ESO llevan entre 1 y 4 años en el centro, el nivel socioeconómico de las familias no es tan alto como los de Primaria y tienen menos asimilada la cultura de centro. 
El alumnado de la muestra se organiza en 9 grupos, siendo la media de edad de 13.95 años, la moda de 15 años y el $38.8 \%$ son chicas.

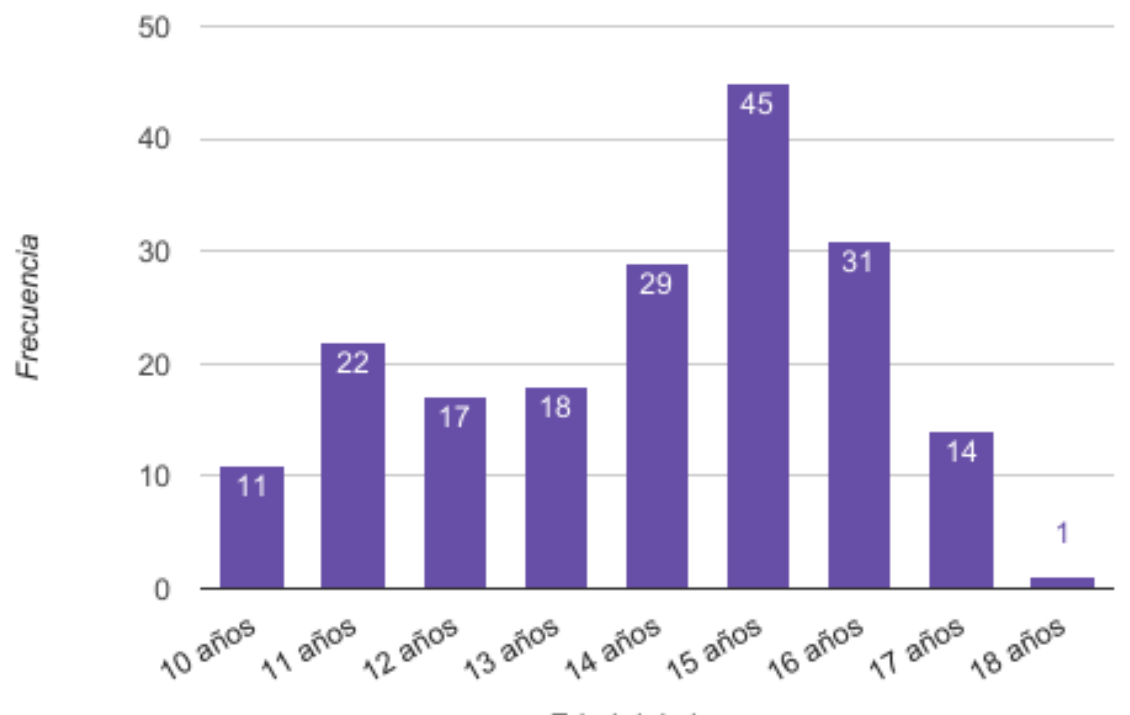

Edad del alumno

Figura 1. Distribución de alumnos por edad

\subsection{INSTRUMENTO DE RECOGIDA DE INFORMACIÓN}

El instrumento de recogida de información que hemos utilizado es un cuestionario ad hoc validado en dos fases y que se administró de manera presencial. Tomamos esta decisión porque el cuestionario es un instrumento que a priori asegura una tasa de respuesta media (en nuestro caso fue total), su coste de elaboración y administración es medio si se utilizan recolectores (en este caso fue bajo, ya que fueron los propios docentes del centro educativo los que lo administraron), la rapidez de administración es media, permite una alta profundidad de los datos y nos permite elaborar el cuestionario de cualquier tamaño razonable (Hernández et al., 2006).

Para elaborar el cuestionario utilizamos el modelo teórico de Castañeda y Adell (2013), quienes a su vez se basaron en Wheeler (2009), y es por ello que seleccionamos seis categorías de análisis: a) Fuentes de información; b) Herramientas; c) Mecanismos; d) Actividades; e) Actitudes y f) Aptitudes. El cuestionario está dividido en tres partes:

- La primera sobre datos demográficos del individuo;

- La segunda se centra en recopilar información sobre uso de dispositivos electrónicos;

- En la tercera recogemos información acerca de cada una de las tres partes que forman el PLE según las categorías elegidas (busco, leo, miro y escucho, dimensión 1; pienso y hago, que denominamos dimensión 2; y, en tercer lugar, hablo, opino y comparto, calificada como dimensión 3).

La validación del cuestionario se llevó a cabo en dos fases. La primera versión del cuestionario fue sometida a juicio de expertos por las dos coordinadoras de etapa y la psicóloga del centro. Como segunda forma de validación elegimos la prueba piloto. Para la misma se seleccionó al grupo de 5. de Primaria por ser estos los alumnos más jóvenes y los que, en principio, podrían tener más dificultades para entender y responder al cuestionario. La prueba se administró de forma presencial a los 26 alumnos de este grupo. Uno de los investigadores estuvo presente para así poder observar y documentar cualquier posible dificultad. Los estudiantes entendieron sin problemas las preguntas y se comprobó que el 
tiempo que habíamos previsto para la administración del cuestionario era suficiente. De esta manera consideramos el instrumento apto para su utilización y utilizamos esta versión para el resto de grupos del centro. La figura 2 muestra el proceso de validación aquí descrito.

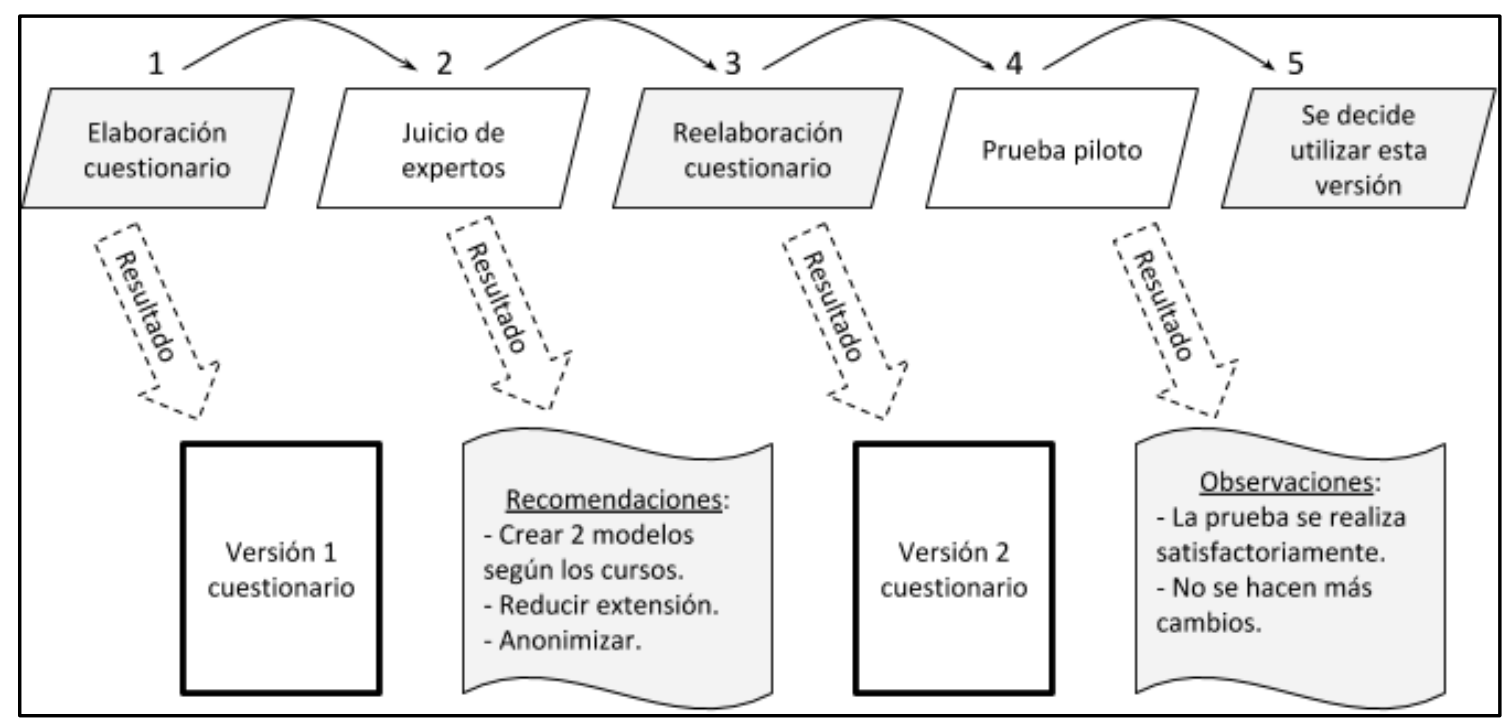

Figura 2. Proceso de validación del cuestionario

\section{RESULTADOS}

En primer lugar y en relación a los dispositivos tecnológicos que usan, el ordenador y el teléfono móvil son los dispositivos que los alumnos dicen poseer con mayor frecuencia $\left(f_{i}=168\right.$ y $f_{i}=164$ respectivamente). A menudo el ordenador es compartido ( $\left.f_{i}=82\right)$, no así el móvil ( $\left.f_{i}=8\right)$. Casi un tercio de los individuos dicen no tener acceso a ningún tipo de tableta ( $\left.f_{i}=56\right)$. Por último, más del $90 \%$ de los alumnos dice tener cuenta de correo electrónico.

Tabla 1. Posesión de dispositivos electrónicos y cuenta de e-mail por parte de los alumnos.

\begin{tabular}{ccccccccc} 
& \multicolumn{2}{c}{ Teléfono móvil } & \multicolumn{2}{c}{ Tableta } & \multicolumn{2}{c}{ Ordenador } & \multicolumn{2}{c}{ e-mail } \\
\cline { 2 - 9 } & $\mathbf{f}_{\mathbf{i}}$ & $\%$ & $\mathbf{f}_{\mathbf{i}}$ & $\%$ & $\mathbf{f}_{\mathbf{i}}$ & $\%$ & $\mathbf{f}_{\mathbf{i}}$ & $\%$ \\
\hline Datos perdidos & 0 & 0 & 1 & 0.5 & 1 & 0.5 & 0 & 0 \\
\hline Propio & 156 & 83 & 61 & 32.4 & 86 & 45.7 & & 91 \\
\hline Compartido & 8 & 4.3 & 70 & 37.2 & 82 & 43.6 & & 91 \\
\hline No & 24 & 12.8 & 56 & 29.8 & 19 & 10.1 & 17 & 9 \\
\hline
\end{tabular}

A continuación nos adentramos en la descripción que hacen de su PLE. Presentamos los resultados en frecuencia media para facilitar la comparación de resultados de diferentes grupos entre sí.

En términos generales, la dimensión 1 (busco y leo) muestra una frecuencia media ligeramente superior a las otras dos de hacer y compartir $\left(f_{m}=4.64\right.$, frente a $f_{m}=4.25$ y $\left.f_{m}=4.19\right)$. El grupo con una mayor cantidad de categorías y subcategorías detectadas en sus cuestionarios es 1.ㅇ de ESO $\left(f_{m}=14.61\right)$ seguido de $3 . \circ$ de ESO $\left(f_{m}=13.74\right)$. Por el contrario, el grupo con una menor frecuencia media es 5. de Primaria $\left(f_{m}=11.96\right)$, seguido de 6. de Primaria $\left(\mathrm{f}_{\mathrm{m}}=12.07\right)$. 
Tabla 2. Componentes del PLE de los alumnos (frecuencia media)

\begin{tabular}{ccccc}
\cline { 2 - 5 } & $\begin{array}{c}\text { Dimensión 1 } \\
\text { busco, leo, miro, } \\
\text { escucho }\end{array}$ & $\begin{array}{c}\text { Dimensión 2 } \\
\text { pienso y hago }\end{array}$ & $\begin{array}{c}\text { Dimensión 3 } \\
\text { hablo, opino, } \\
\text { comparto }\end{array}$ & Total \\
\hline $5 .^{\circ}$ Primaria & 4.35 & 3.65 & 3.96 & 11.96 \\
\hline $6 .^{\circ}$ Primaria & 4.67 & 3.33 & 4.07 & 12.07 \\
\hline $1 .^{\circ}$ ESO & 5.38 & 3.71 & 5.52 & 14.61 \\
\hline $2 .^{\circ}$ ESO & 3.65 & 5.43 & 4.22 & 13.3 \\
\hline $3 .^{\circ}$ ESO & 5.11 & 4.6 & 4.03 & 13.74 \\
\hline $4 .^{\circ}$ ESO & 4.66 & 4.74 & 3.32 & 13.08 \\
\hline Global & 4.64 & 4.25 & 4.19 & 132 \\
\hline
\end{tabular}

\section{Componentes del PLE (frecuencia media)}

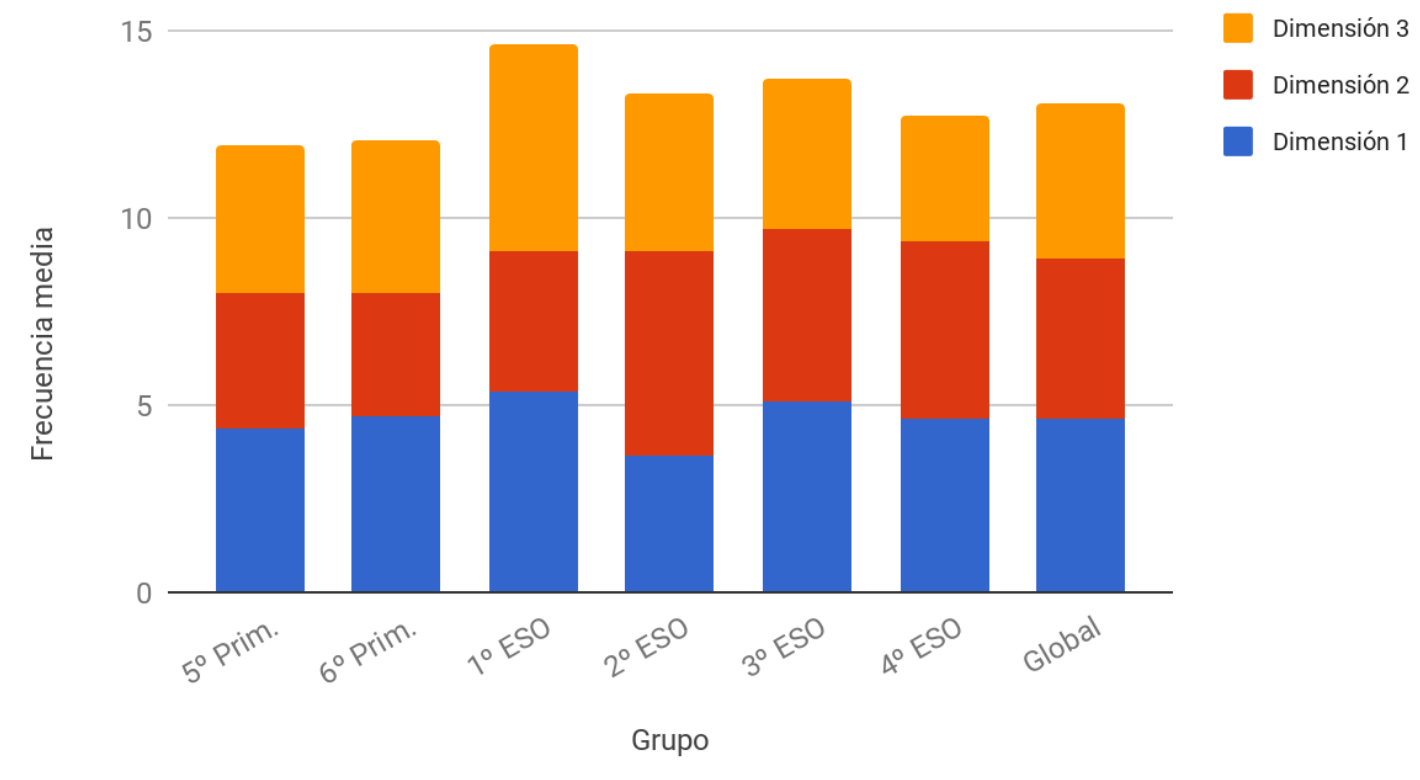

Figura 3. Componentes del PLE de los alumnos (frecuencia media)

En la figura 4 presentamos los resultados de forma que podamos comparar las diferentes dimensiones del PLE dentro de un mismo grupo y de los diferentes grupos entre sí. La dimensión 1 (busco, leo, miro y escucho) está más desarrollada en los grupos de 1. de ESO $\left(f_{m}=5.38\right)$ y $3 . . .0$ de ESO $\left(f_{m}=5.11\right)$ y notablemente menos desarrollada en $2 . . \circ$ de ESO $\left(f_{m}=3.65\right)$. La dimensión 2 (pienso y hago) destaca en $2 . .0$ de ESO $\left(f_{m}=5.43\right)$ y $4 . . .0$ de ESO $\left(f_{m}=4.74\right)$ y por el contrario está menos presente en los cuestionarios de los alumnos de 6. y 5.. de Primaria ( $\mathrm{f}_{\mathrm{m}}=3.33$ y $\mathrm{f}_{\mathrm{m}}=3.65$, respectivamente). Por último, la dimensión 3 (hablo, opino y comparto) presenta una frecuencia media sensiblemente más elevada en el grupo de 1. de ESO $\left(f_{m}=5.52\right)$ y menos desarrollada en $4 . . .0$ de ESO $\left(f_{m}=3.32\right)$. 


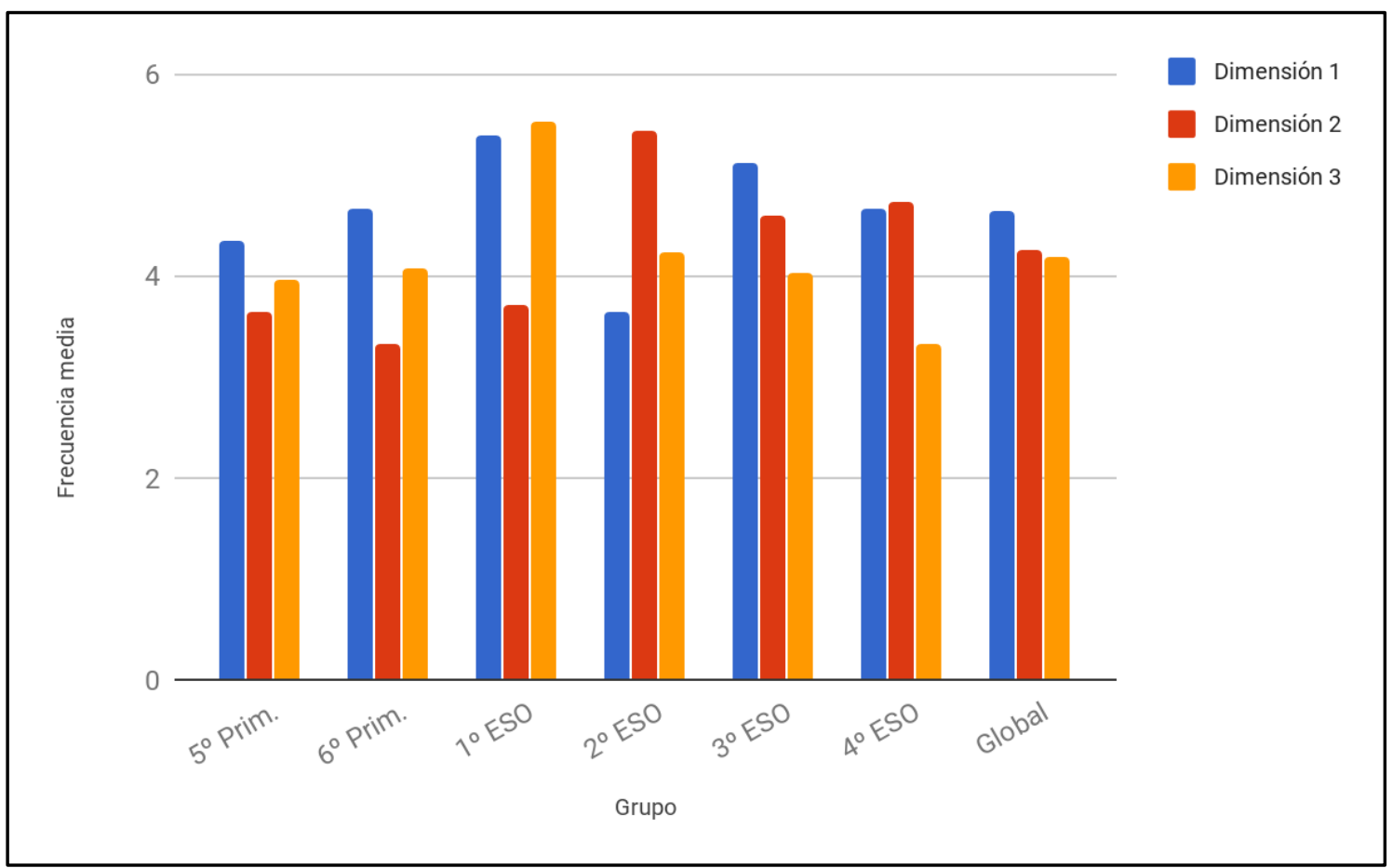

Figura 4. Componentes del PLE de los alumnos (frecuencia media)

A continuación, pasamos a presentar los datos de las categorías detectadas para cada una de las partes del PLE de la muestra analizada. Hemos eliminado las categorías de Actitudes y Aptitudes por su escasa relevancia en nuestra investigación ( $f_{m}=0.14$ y $f_{m}=0.32$ respectivamente para las tres dimensiones estudiadas).

La tabla 3 y la figura 5 muestran la información referente a la dimensión 1 . En ella los grupos donde más categorías se han detectado son $1 .$. y $3 .$. de $E S O\left(f_{m}=5.00\right.$ y $f_{m}=4.91$ respectivamente); en 2.. de ESO por el contrario se ha detectado el menor número de categorías $\left(f_{m}=3.39\right)$. La categoría Herramientas es la más abundante $\left(f_{m}=2.03\right)$, muy por encima de Fuentes de información $\left(f_{m}=0.89\right)$, Mecanismos $\left(f_{m}=0.78\right)$ y Actividades $\left(f_{m}=0.67\right)$.

Tabla 3. Categorías detectadas en la parte 1 (busco, leo, miro, escucho) del PLE de los alumnos (frecuencia media)

\begin{tabular}{cccccc} 
& $\begin{array}{c}\text { Fuentes de } \\
\text { información }\end{array}$ & Herramientas & Mecanismos & Actividades & Total \\
\hline $5 .^{\circ}$ Primaria & 0.58 & 2.27 & 0.85 & 0.42 & 4.12 \\
\hline $6 .^{\circ}$ Primaria & 1.07 & 1.87 & 0.73 & 0.60 & 4.27 \\
\hline $1 .^{\circ} \mathrm{ESO}$ & 0.9 & 2.29 & 0.86 & 0.95 & 5.00 \\
\hline $2 .^{\circ} \mathrm{ESO}$ & 0.57 & 1.65 & 0.52 & 0.65 & 3.39 \\
\hline $3 .^{\circ} \mathrm{ESO}$ & 1.27 & 2.17 & 0.62 & 0.85 & 4.91 \\
\hline $4 .^{\circ} \mathrm{ESO}$ & 0.94 & 1.91 & 1.10 & 0.51 & 4.46 \\
\hline Global & $\mathbf{0 . 8 9}$ & $\mathbf{2 . 0 3}$ & $\mathbf{0 . 7 8}$ & $\mathbf{0 . 6 7}$ & $\mathbf{4 . 3 7}$ \\
\hline
\end{tabular}




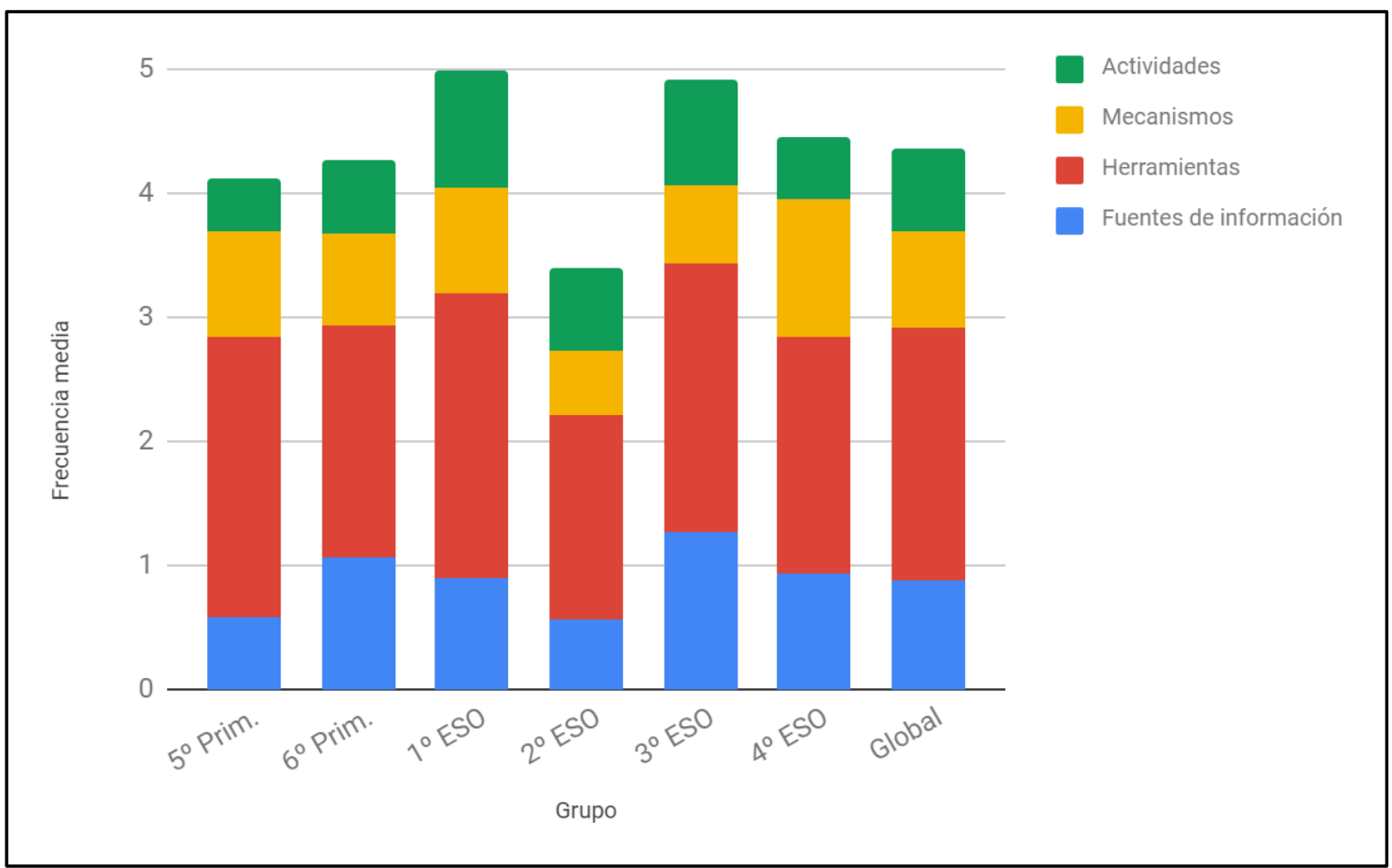

Figura 5. Categorías detectadas en la parte 1 (busco, leo, miro, escucho) del PLE de los alumnos (frecuencia media)

La tabla 4 y la figura 6 muestran la información referente a la dimensión 2. Aquí son los propios alumnos los que hacen la función de fuente de información; por este motivo no se ha detectado esta categoría en los cuestionarios analizados. El grupo donde más categorías se han detectado es $2 .$. o de $\mathrm{ESO}\left(\mathrm{f}_{\mathrm{m}}=5.39\right)$, y el que menos 6.. de Primaria $\left(\mathrm{f}_{\mathrm{m}}=3.26\right)$. La categoría más abundante es Herramientas ( $\left.f_{m}=2.55\right)$, y la que menos Mecanismos $\left(f_{m}=0.39\right)$.

Tabla 4. Categorías detectadas en la parte 2 (pienso y hago) del PLE de los alumnos (frecuencia media)

\begin{tabular}{cccccc}
\cline { 2 - 6 } & $\begin{array}{c}\text { Fuentes de } \\
\text { información }\end{array}$ & Herramientas & Mecanismos & Actividades & Total \\
\hline $5 .{ }^{\circ}$ Primaria & 0 & 2.69 & 0.42 & 0.42 & 3.53 \\
\hline $6 .{ }^{\circ}$ Primaria & 0 & 2.33 & 0.40 & 0.53 & 3.26 \\
\hline $1 .^{\circ} \mathrm{ESO}$ & 0 & 1.90 & 0.33 & 1.43 & 3.66 \\
\hline $2 .^{\circ} \mathrm{ESO}$ & 0 & 3.04 & 0.35 & 2.00 & 5.39 \\
\hline $3 .^{\circ} \mathrm{ESO}$ & 0 & 2.70 & 0.34 & 1.45 & 4.49 \\
\hline $4 .^{\circ} \mathrm{ESO}$ & 0 & 2.65 & 0.48 & 1.59 & 4.72 \\
\hline Global & $\mathbf{0}$ & $\mathbf{2 . 5 5}$ & $\mathbf{0 . 3 9}$ & $\mathbf{1 . 2 4}$ & $\mathbf{4 . 1 8}$ \\
\hline
\end{tabular}




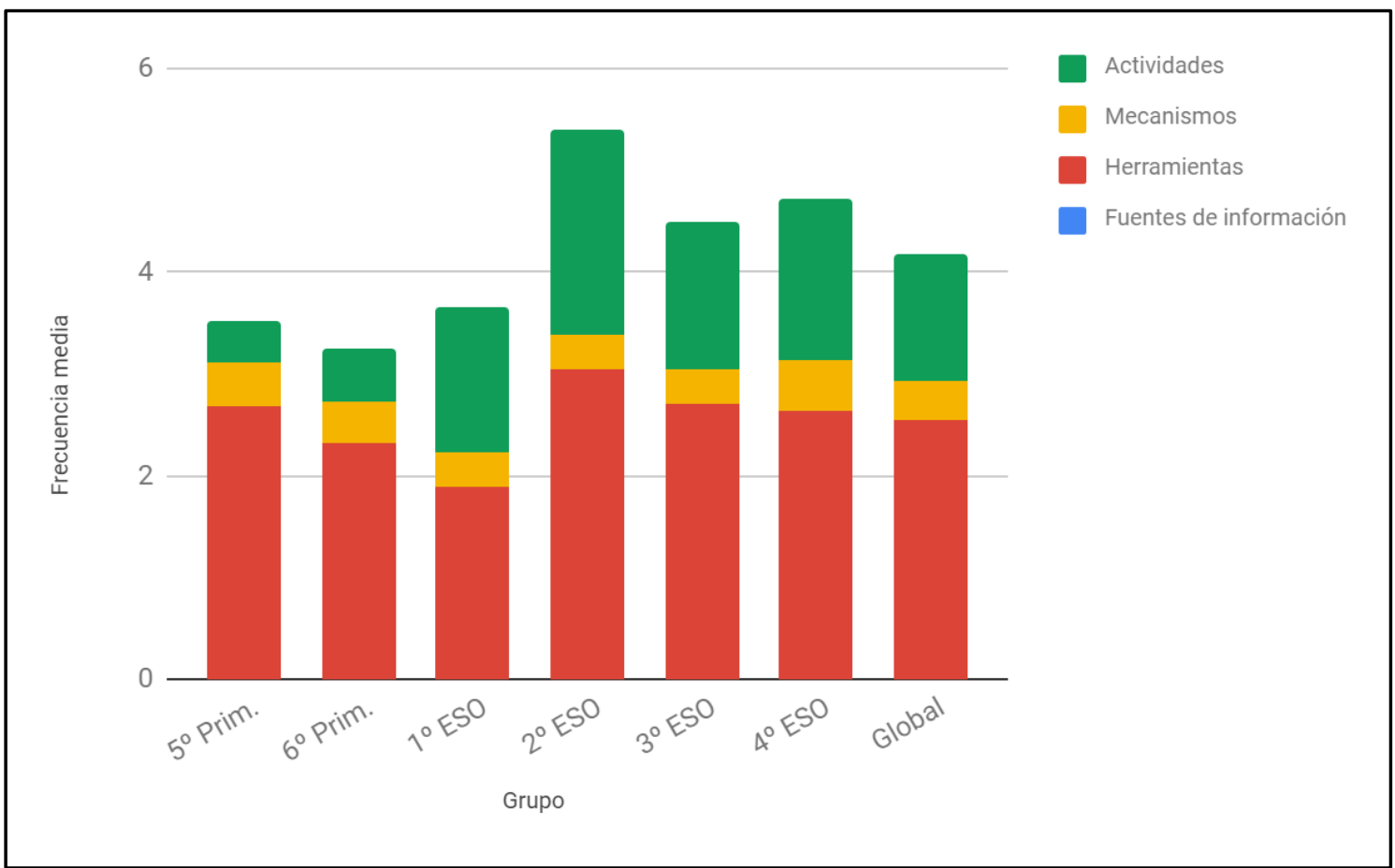

Figura 6. Categorías detectadas en la parte 2 (pienso y hago) del PLE de los alumnos (frecuencia media)

Por último, la tabla 5 y la figura 7 muestran la información referente a la dimensión 3 , y nos permiten observar que $1 .$. de ESO es el grupo con mayor cantidad de categorías detectadas $\left(f_{m}=5.19\right)$, y $4 .{ }^{\circ}$ de $E S O$ el que menos $\left(f_{m}=3.24\right)$. La categoría más abundante es una vez más Herramientas $\left(f_{m}=2.01\right)$, y las que menos presencia tienen son Fuentes de información y Mecanismos (respectivamente $\mathrm{f}_{\mathrm{m}}=0.20$ y $\mathrm{f}_{\mathrm{m}}=0.21$ ).

Tabla 5. Categorías detectadas en la parte 3 (hablo, opino, comparto) del PLE de los alumnos (frecuencia media)

\begin{tabular}{cccccc}
\cline { 2 - 6 } & $\begin{array}{c}\text { Fuentes de } \\
\text { información }\end{array}$ & Herramientas & Mecanismos & Actividades & Total \\
\hline $5 .{ }^{\circ}$ Primaria & 0.04 & 1.96 & 0 & 1.96 & 3.96 \\
\hline $6 .{ }^{\circ}$ Primaria & 0.33 & 1.87 & 0.27 & 1.60 & 4.07 \\
\hline $1 .{ }^{\circ}$ ESO & 0.43 & 1.95 & 0.57 & 2.24 & 5.19 \\
\hline $2 .^{\circ}$ ESO & 0.17 & 2.00 & 0.30 & 1.61 & 4.08 \\
\hline $3 .{ }^{\circ}$ ESO & 0.11 & 2.33 & 0.06 & 1.43 & 3.93 \\
\hline $4 .{ }^{\circ}$ ESO & 0.12 & 1.94 & 0.04 & 1.14 & 3.24 \\
\hline Global & $\mathbf{0 . 2 0}$ & $\mathbf{2 . 0 1}$ & $\mathbf{0 . 2 1}$ & $\mathbf{1 . 6 6}$ & $\mathbf{4 . 0 8}$ \\
\hline
\end{tabular}




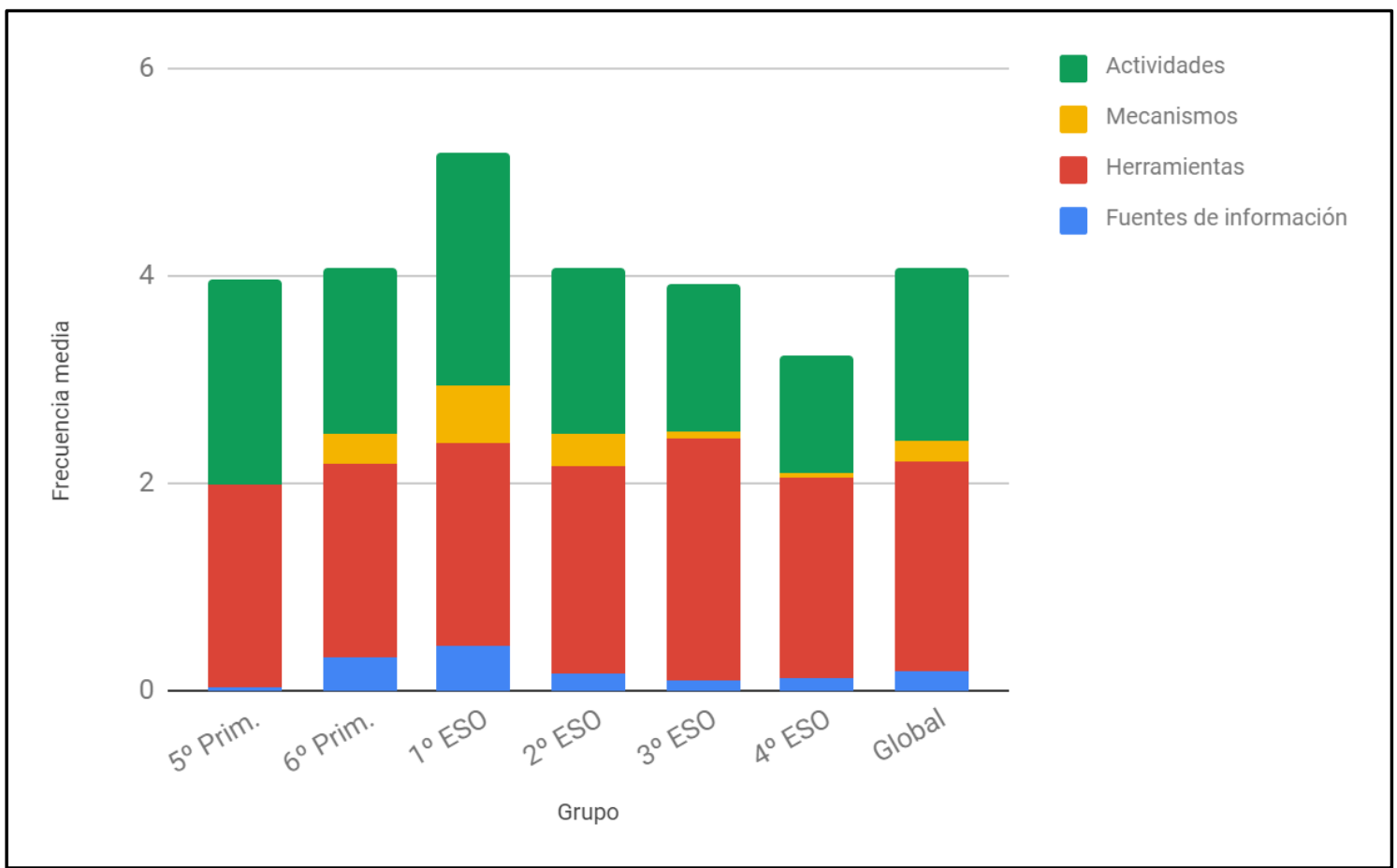

Figura 7. Categorías detectadas en la parte 3 (hablo, opino, comparto) del PLE de los alumnos (frecuencia media)

\section{CONCLUSIONES}

Las dimensiones que conforman el PLE de los alumnos analizados presentan una cantidad de categorías y subcategorías muy similar. Esto se puede interpretar como que los alumnos demuestran el mismo nivel de competencia al buscar información, al crearla y al compartirla en red. En los cuestionarios se detectan categorías y subcategorías en cantidades bajas, algo que nunca se debe achacar a la falta de acceso a dispositivos electrónicos puesto que la muestra analizada admite tener fácil acceso a dispositivos que además son de gama alta. Las categorías que se detectan con mayor frecuencia son Herramientas, Actividades y Fuentes de información (por ese orden), y las que menos Mecanismos, Actitudes y Aptitudes.

Los alumnos de ESO muestran generalmente PLEs ligeramente más ricos que los de Primaria. Se trata de alumnos mayores a los que se les presupone mayor experiencia académica, mayor bagaje en términos de aprendizaje y niveles más altos de desarrollo madurativo. Pero siguiendo este razonamiento, el curso con un PLE más enriquecido debería ser 4..$^{\circ}$ de ESO -y no 1. de ESO como sucede realmente-. La diferencia entre unos alumnos y otros es poco significativa, lo que nos debe llevar a plantearnos cómo por una parte los alumnos más jóvenes pueden estar igual de preparados que los mayores para recibir formación avanzada que desarrolle su competencia tecnológica y cómo, por otro lado, los alumnos mayores a menudo no aprovechan al máximo los recursos a su disposición.

Si entramos a analizar cada grupo por separado, los grupos de $5 .^{\circ}$ y $6 .^{\circ}$ de Primaria demuestran manejar bien todo lo relacionado con la búsqueda y lectura de información, pero presentan deficiencias al procesarla y crear artefactos en red. Los alumnos de 1. de ESO conocen y utilizan bien las herramientas de lectura y organización de la información y muestran buenos niveles de competencia para la socialización y el compartir en red a través de su PLN, pero necesitan trabajar todo lo relacionado con la reflexión y producción en red. Justo al contrario ocurre con $2 .^{\circ}$ de ESO, quienes muestran conocer bien las herramientas y actividades para la reflexión y creación en red, pero necesitan aprender más sobre cómo consumir y organizar la información, así como sobre relacionarse con otras personas a través de la red con fines de aprendizaje.

3. de ESO y $4 . .^{\circ}$ de ESO muestran cierto equilibrio entre las partes, aunque en $4 . .^{\circ}$ de ESO llama la atención la poca cantidad de categorías y subcategorías detectadas en la dimensión 3. 
Son los alumnos mayores, teóricamente más independientes y autónomos en su aprendizaje pero que todavía necesitan adquirir competencias para la reflexión y creación en red y, sobre todo, para la socialización y las estrategias para compartir la información.

\section{DISCUSIÓN}

Nuestra investigación se asemeja a la realizada por Ricoy y Couto (2010), estudio igualmente descriptivo -aunque desde un enfoque cualitativo- que se desarrolla en la zona norte de Portugal y donde se recoge información sobre factores que rodean al uso de las TIC en Secundaria. El resto de proyectos que hemos analizado son de tipo investigación-acción (Döbeli \& Neff, 2010; Martín \& Torres, 2010; Ricoy \& Couto, 2010; Rahimi et al., 2012; Pais et al., 2013; Cabero, 2014; Usaquén, 2015; Gil et al., 2016; Nieto \& Dondarza, 2016; Prendes et al., 2016; Restrepo, 2016; Vázquez et al., 2016). Este tipo de investigaciones requieren más tiempo y esfuerzo por parte de todos los actores involucrados, por ejemplo, para trabajar sobre el PLE de los propios docentes, ya que son ellos quienes tienen que aplicar con sus alumnos en última instancia las intervenciones sugeridas. Aunque en nuestro trabajo no hemos recogido información sobre el PLE de los docentes, sí hemos podido observar ciertas diferencias entre el PLE de diferentes grupos de estudiantes que apuntan a éste como un factor muy importante a la hora de determinar el nivel de calidad del PLE de los mismos.

Hay diferencias importantes entre los diferentes proyectos en cuanto a facilidad de acceso a dispositivos tecnológicos y tipología de los mismos. Existe un primer grupo de investigaciones (Döbeli \& Neff, 2010; Rahimi et al., 2012; Nieto \& Dondarza, 2016) en las que los alumnos tenían fácil acceso a los dispositivos. En otro segundo grupo de trabajos (Martín \& Torres, 2010; Ricoy \& Couto, 2010; Pais et al., 2013; Usaquén, 2015; Gil et al., 2016; Restrepo, 2016) no se pone el foco sobre los dispositivos que los alumnos y profesores van a utilizar, sino más bien en los programas, aplicaciones y recursos en red. Nuestro trabajo podría encuadrarse en el primer grupo, ya que hemos encontrado abundancia de recursos y estos son por lo general de alta calidad.

La cantidad y no la calidad de los dispositivos utilizados parece ser un factor determinante a la hora de definir si un proyecto es exitoso o no, ya que la falta de recursos o la dificultad para solucionar problemas técnicos pueden llevar a que se genere una sensación de frustración entre los participantes, como se podía observar en Rahimi et al. (2012) y Gil et al. (2016).

En relación a la edad de los sujetos objeto de estudio, nuestro trabajo coincide con Pais et al. (2013), ya que como ellos hemos analizado el PLE de alumnos de las etapas de Primaria y Secundaria. La conclusión a la que llegamos es la misma a la que llegan el resto de autores en su conjunto (Döbeli \& Neff, 2010; Martín \& Torres, 2010; Ricoy \& Couto, 2010; Rahimi et al., 2012; Usaquén, 2015; Gil et al., 2016; Nieto \& Dondarza, 2016; Restrepo, 2016), y es que la edad del alumno no tiene por qué ser un problema a la hora de trabajar el PLE del propio alumno. Coincidimos con todos estos autores en detectar abundancia de información sobre las categorías de Herramientas, Actividades y Fuentes y carencia de Mecanismos, Actitudes y Aptitudes.

Por ejemplo, nuestros alumnos de $4 .^{\circ}$ de ESO tienen una red personal de aprendizaje mucho más pobre que la de los alumnos de $1 .$. de ESO; y los alumnos de $5 .^{\circ}$ de Primaria citan muchas más herramientas de lectura que los de 2. de ESO, cuando en principio esperábamos que ocurriera lo contrario. De hecho, conceptos que los autores citados anteriormente consideran de vital importancia para sus proyectos como la autorregulación, la autonomía, la identidad o la independencia se trabajan de manera exitosa con alumnos de ambas etapas educativas. En cualquier caso, es obligación del docente y del centro educativo adaptar las tareas al nivel de desarrollo psicológico y cognitivo del alumno.

Adentrándonos en otro aspecto, cabría pensar que los alumnos se sienten más cómodos utilizando las herramientas tecnológicas disponibles mediante metodologías activas, pero a veces esto no es así. En Döbeli \& Neff (2010) y Nieto \& Dondarza (2016) se habla explícitamente de que los alumnos prefieren formas de trabajo tradicionales, con o sin tecnología, algo que coincide con la opinión de alumnos universitarios en Prendes et al. (2016). Una posible explicación para esto es que con las formas de trabajo tradicionales los alumnos 
tenían un rol pasivo, probablemente aburrido pero cómodo a la vez. En una metodología de trabajo activa (y más aún bajo la idea de PLE) el alumno es el que dirige su propio aprendizaje, algo que no está acostumbrado a hacer y que, por tanto, requiere de él mayor esfuerzo. Los datos de nuestro estudio apuntan también en esta dirección, porque aunque no tenemos información específica sobre las preferencias de los alumnos en cuanto a metodologías de enseñanza ni a estilos de aprendizaje, los datos descriptivos del PLE que hemos obtenido muestran que las categorías y subcategorías detectadas con mayor frecuencia encajan cómodamente en un entorno de enseñanza tradicional.

Por último, en las experiencias analizadas los investigadores parecen dar preferencia al trabajo con la dimensión 2 del PLE de sus alumnos (Martín \& Torres, 2010; Rahimi et al., 2012), seguido de la dimensión 3 (Pais et al., 2013; Gil et al., 2016). Döbeli \& Neff (2010) destaca como experiencia que da especial énfasis al trabajo de la dimensión 1 del PLE. Esto coincide con lo que nos hemos encontrado en nuestro estudio: el PLE de los alumnos analizados está relativamente equilibrado, pero aún así las partes más pobres son la parte 3 y la parte 2 en este orden, siendo la parte 1 la más rica a priori y, por consiguiente, la que menos urgencia tiene para ser trabajada.

\section{RECOMENDACIONES}

Los resultados de la investigación que hemos presentado en este artículo parecen indicar que hay ciertos aspectos en torno al PLE de los alumnos analizados que conviene reforzar. Esto se debe hacer mediante un plan de formación del profesorado que deberá ser propuesto por parte del Coordinador TIC y del equipo docente (según el contexto del centro, puede ser un plan diseñado con la colaboración del equipo de orientación y/o el equipo directivo). En la tabla 3 se muestran los temas que deben ser impartidos de forma más urgente, bien por la importancia del tema, bien por el nivel de desconocimiento que los alumnos han demostrado sobre el mismo. No hemos incluido ninguna propuesta formativa referente a las categorías de actitudes y aptitudes porque consideramos que la formación en estos aspectos se debe dar de manera transversal a través del resto de actividades académicas.

En la tabla tampoco se han incluido algunos de los aspectos críticos para el éxito de un proyecto de este tipo; la autonomía, la autorregulación o la identidad digital se tratarán de manera indirecta en los módulos formativos propuestos de seguridad, netiqueta y compartición en red.

Tabla 6. Propuesta de plan de actuación para el centro

\begin{tabular}{|c|c|c|c|}
\hline Partes del PLE & Categoría & Nombre & Descripción \\
\hline \multirow{3}{*}{ Todas } & \multirow{3}{*}{ Todas } & PLE & $\begin{array}{l}\text { Concepto, importancia y formas de representación, } \\
\text { aplicación, evaluación y mejora del propio PLE. }\end{array}$ \\
\hline & & mLearning & $\begin{array}{l}\text { Competencia instrumental en el uso de móviles y tablets } \\
\text { para el aprendizaje. }\end{array}$ \\
\hline & & Seguridad & $\begin{array}{l}\text { Utilización segura y eficiente de servicios y contraseñas en } \\
\text { red. }\end{array}$ \\
\hline \multirow{3}{*}{ Dimensión 1} & $\begin{array}{l}\text { Fuentes, } \\
\text { Mecanismos }\end{array}$ & $\begin{array}{l}\text { Capacidad } \\
\text { crítica }\end{array}$ & $\begin{array}{l}\text { Búsqueda y selección de información fiable y relevante en } \\
\text { variedad de fuentes diferentes. }\end{array}$ \\
\hline & Herramientas & Organización & Gestión y ordenación de la información en la nube. \\
\hline & Actividades & $\begin{array}{l}\text { Lectura } \\
\text { multimedia }\end{array}$ & Lectura de información en diferentes formatos. \\
\hline Dimensión 2 & Herramientas & $\begin{array}{l}\text { Creación } \\
\text { multimedia }\end{array}$ & $\begin{array}{l}\text { Competencia instrumental en el uso de herramientas para } \\
\text { la creación multimedia y fomento de su uso como medio }\end{array}$ \\
\hline
\end{tabular}


de expresión (alta necesidad en Primaria).

Herramientas Compartición Selección y uso de herramientas para compartir en red información en red.

\begin{tabular}{llll}
\hline Dim. 2 y 3 & Todas & Seguridad & $\begin{array}{l}\text { Prevención de situaciones de riesgo al compartir o } \\
\text { publicar información y al interactuar con otras personas en } \\
\text { red. }\end{array}$ \\
\hline & Todas & PLN & $\begin{array}{l}\text { Concepto, importancia y formas de representación, } \\
\text { evaluación y mejora de la propia PLN. }\end{array}$ \\
\cline { 2 - 4 } Dimensión 3 & Herramientas & PLN & $\begin{array}{l}\text { Conocimiento de herramientas variadas para la gestión y } \\
\text { utilización de la propia PLN. }\end{array}$ \\
\cline { 2 - 4 } & Todas & Netiqueta & Normas de conducta deseables al socializar en red. \\
\hline
\end{tabular}

De cara al futuro sería interesante replicar esta investigación en otros contextos, así como llevar a cabo nuevas investigaciones desde un enfoque mixto o con combinación de enfoques, pues eso enriquecería los datos recogidos y la fiabilidad de los resultados.

Para finalizar, esperamos que este trabajo sirva para arrojar algo de luz al tema de los Entornos Personales de Aprendizaje y ayude a ampliar la literatura sobre experiencias con PLE de alumnos reales y en contextos de aprendizaje específicos.

\section{ENLACES}

Página oficial del Máster Interuniversitario en Tecnología Educativa: e-learning y Gestión del Conocimiento: https://www.um.es/web/educacion/contenido/estudios/masteres/e-learning

Instrumento de recogida de información (5. Primaria - 2.ำESO) (elaboración propia): https://drive.google.com/open?id=1ADesPGPsYfrGZvBqBHs6aQ4FOq80cXJeiQN90|3w9uk

Instrumento de recogida de información (3. ESO - 4. ESO) (elaboración propia): https://drive.google.com/open?id=15FDYxMFo5nMEG5tDUulwGfkcw78wSWKB3GvX4HT8IKY

Trabajo Fin de Máster del cual se ha extraído este artículo (Jiménez, 2017): https://drive.google.com/file/d/0BxRB ob5QKPsWjVlckFObm5pVU0/view?usp=sharing

\section{REFERENCIAS BIBLIOGRÁFICAS}

Attwell, G. (2007). Personal Learning Environments-the future of eLearning? eLearning papers, 2(1), 1-8. https://bit.ly/2JGtSi5

Benito, M. (2016, November 2). Investigando PLEs [Video]. TV.um.es. https://bit.ly/2RcxY5L

Cabero, J. (2014). Creación de entornos personales de aprendizaje como recurso para la formación. El proyecto Dipro 2.0. EDUTEC, Revista Electrónica de Tecnología Educativa, 47. https://bit.ly/2VOYEri

Castañeda, L. \& Adell, J. (Eds.) (2013). Entornos Personales de Aprendizaje: Claves para el ecosistema educativo en red (pp. 11-27). Marfil.

Castañeda, L., Cosgrave, M. \& Marín, V. (2016). Personal Learning Environments: PLE Conference 2015 Special Issue Guest Editorial. Digital Education Review, 29. https://bit.ly/2UHjoW3

Dabbagh, N., \& Kitsantas, A. (2011). Personal learning environments, social media and selfregulated learning: A natural formula for connecting formal and informal learning. Internet and Higher Education, 15, 3-8. https://doi.org/10.1016/.j.iheduc.2011.06.002 
Döbeli, B. \& Neff, C. (2010). Personal Smartphones in Primary School: Devices for a PLE? [Conference]. The PLE Conference 2010, Barcelona, Spain. https://bit.ly/39F4kwp

Hernández, R., Fernández, C., \& Baptista, P. (2006). Metodología de la investigación. McGrawHill.

Martín, R. \& Torres, R. (2010). Ventajas pedagógicas en la aplicación del PLE en asignaturas de lengua y literatura de educación secundaria. Análisis de cinco experiencias [Conference]. The PLE Conference 2010, Barcelona, Spain. https://bit.ly/3aDVMqZ

Milligan, C. (2006). The road to the personal learning environment. https://bit.ly/3dUsal4

Nieto, E. \& Dondarza, P. (2016). PleS in Primary School: The Learners' experience in the Piplep Project. Digital Education Review, 29, 45-61. https://bit.ly/39F4vb3

Pais, F., Santos, C. \& Pedro, L. (2013). Innovation, knowledge and sustainability with PLEs: an empirical analysis from SAPO Campus Schools pilots. In Buchem, I., Attwell, G. \& Tur, G. (Eds.), The PLE Conference 2013 Proceedings (pp. 113-133). Beuth University of Applied Sciences \& Monash University. https://bit.ly/2JBFeUv

Peña, I. (2013). El PLE de investigación-docencia: el aprendizaje como enseñanza. In L. Castañeda \& J. Adell (Eds.), Entornos Personales de Aprendizaje: Claves para el ecosistema educativo en red (pp. 93-110). Marfil. https://bit.ly/3bRTAMS

Prendes, M.P., Castañeda, L., Gutiérrez \& Román, M. (2016). Still far from Personal Learning: Key aspects and Emergent topics about How future Professionals' PLEs are. Digital Education Review, 29, 15-30. https://bit.ly/2yu0Obi

Prendes, M.P. \& Román, M. (coords.) (2017). Entornos Personales de Aprendizaje: una visión actual de cómo aprender con tecnologías. Octaedro.

Prendes, M.P., Román, M.M. \& González, V. (2019). How University Students Use Technologies to Learn: A Survey about PLE in Spain. Education in the knowledge society, 20, 1-10. https://dx.doi.org/10.14201/eks2019 20 a10

Rahimi, E., van den Berg, J. \& Veen, W. (2012). Designing and Implementing PLEs in a Secondary School Using Web2.0 Tools [Conference]. The PLE Conference 2012, Aveiro, Portugal \& Melbourne, Australia. https://bit.ly/2JGkE5q

Salinas, J., Marín, V. \& Escandell, C. (2011). A case of institutional PLE: integration of VLE and e-portfolio for students [Conference]. The PLE Conference 2011, Southampton, United Kingdom. https://bit.ly/34aR5Cw

Van Harmelen, M. (2006). Personal Learning Environments. In Kinshuk, Koper, R., Kommers,, P., Kirschner, P., Sampson, D. \& Didderen, W. (Eds.), ICALT 2006 Proceedings (pp. 815-816). https://dx.doi.org/10.1109/ICALT.2006.1652565

Vázquez E., Martín, E. \& Castrillo, M.D. (2016). Analysis of PLEs' Implementation under OER design as a productive teaching-learning strategy in Higher Education. A case study at Universidad Nacional de educación a Distancia. Digital Education Review, 29, 62-85. https://bit.ly/2V2DxVx

Wheeler, S. (2009, October 12). It's Personal: Learning Spaces, Learning Webs. Learning with 'e's. My thoughts about learning technology and all things digital. https://bit.ly/2wReJrH

Wheeler, S. (2012, July 9). What is learning? Learning with 'e's. My thoughts about learning technology and all things digital. https://bit.ly/2JG5bm8 


\title{
INFORMACIÓN SOBRE EL AUTOR
}

\section{Alberto Jiménez Hidalgo}

Universidad de Murcia

Licenciado en Filología Inglesa por la Universidad Complutense de Madrid y Máster en Tecnología Educativa por la Universidad de Murcia, con experiencia docente en Educación Infantil, Primaria y Secundaria en España, Reino Unido y Estados Unidos. El tema central de mi TFM son los Entornos Personales de Aprendizaje (PLE) y cómo estos se manifiestan en contextos de Enseñanza Obligatoria.

Twitter: @MrAlberto_JH

Facebook: https://www.facebook.com/mralberto.jh1$$
\text { (c) (1) (9) }
$$

Los textos publicados en esta revista están sujetos a una licencia de Reconocimiento 4.0 España de Creative Commons. Puede copiarlos, distribuirlos, comunicarlos públicamente y hacer obras derivadas siempre que reconozca los créditos de las obras (autoría, nombre de la revista, institución editora) de la manera especificada por los autores o por la revista. La licencia completa se puede consultar en:Licencia Creative Commons Atribución-NoComercial-Compartir por igual 4.0 Internacional.
\end{abstract}

